\title{
Iatrogener Nasenfremdkörper
}

\author{
J. Ritter, A. Berghaus \\ Universitäts-HNO-Klinik (Leiter: Prof. Dr. H. Scherer) Klinikum Steglitz der Freien Universität Berlin
}

\section{Zusammenfassung}

In einer Kasuistik wird über einen intranasalen Fremdkörper berichtet, der fünf Monate nach konservativer Therapie eines Herzinfarktes entdeckt wurde. Für die Fixierung von Sauerstoffsonden werden dabei Kunststoffblockungen benutzt, die akzidentell in der Nase steckenbleiben können. Im vorliegenden Fall entstand zunächst der Verdacht auf einen Tumor der inneren Nase.

\section{Iatrogenic Intranasal Foreign Body}

The present case involves an intranasal foreign body that was discovered five months after conservative therapy of a myocardial infarction. In such cases, oxygen tubes are fixed by plastic blocks which can accidentally become lodged in the nose. In this case, an elderly patient was suspected of having a carcinoma of the inner nose.

\section{Einleitung}

Nasenfremdkörper findet man vor allem bei Kindern, die sich in Spiellaune bevorzugt Spielzeugteile in die Nase stecken. Beim nicht psychisch gestörten $\mathrm{Er}$ wachsenen sind es überwiegend Rückstände nach Nasenoperationen, wie z. B. Implantate oder Tamponadereste (1, 2), die nach dem Auftreten von Beschwerden meist schnell erkannt und entfernt werden, zumal die Anamnese diagnoseweisend ist. In dem hier zu berichtenden Fall war aber die Applikation des Fremdkörpers zunächst unbekannt, was dazu führte, daß die Beschwerden primär als Symptome eines fraglich bösartigen Tumors der inneren Nase fehlgedeutet wurden.

\section{Kasuistik}

Ein 78jähriger Patient, der im Februar 1987 die HNO-Poliklinik aufsuchte, berichtete über seit Monaten bestehende, einseitig behinderte Nasenatmung mit blutiger, später auch eitriger Sekretion. Aus der Vorgeschichte war ein Herzinfarkt im September 1986 bemerkenswert. Bei der Erstvorstellung wurde neben einer ausgeprägten s-förmigen Septumdeviation endoskopisch auf der rechten Seite eine leicht blutende, nahezu verlegene Gewebeschwellung im unteren Nasengang gesehen. Eine Probeexzision wurde durchgeführt und ein Computertomogramm veranlaßt (Abb. 1).

Laryngo-Rhino-Otol. 68 (1989) 299-300

(c) Georg Thieme Verlag Stuttgart · New York

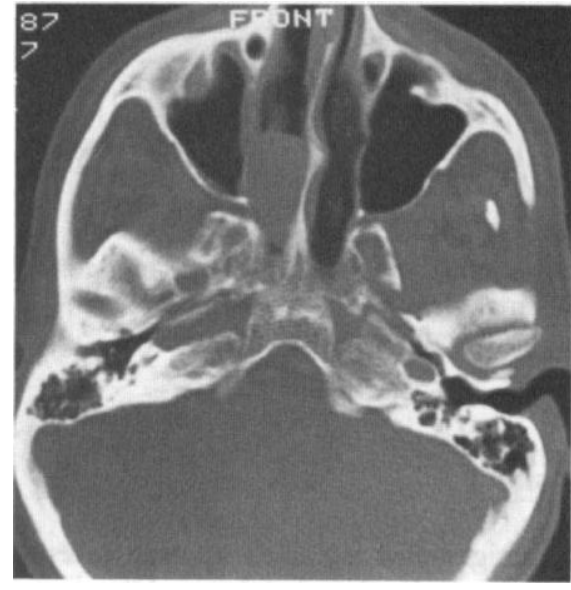

Abb.1 Das Computertomogramm zeigt einen polypoiden Tumor im hinteren Nasenbereich rechts mit gering expansivem Charakter, jedoch ohne Nachweis eines knochendestruierenden Wachstums.

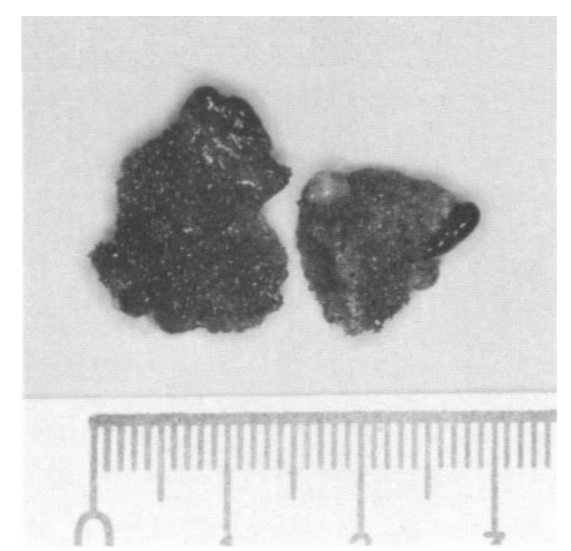

Abb. 2 Der extrahierte Fremdkörper.

Das Computertomogramm ließ einen polypoiden Tumor im hinteren Nasenbereich mit gering expansivem Charakter erkennen, allerdings ohne eindeutige Knochendestruktion. Die histopathologische Begutachtung ergab eine plasmazelluläre Entzündung mit histiozytärer Reaktion um multiple Fremdkörper.

Nach Vorliegen dieser Befunde erfolgte erneut eine ausführliche Nasenendoskopie mit starren Optiken. Dabei wurden Schaumstoffteile extrahiert, wie sie zur Fixierung von Sauerstoffsonden verwendet werden (Abb. 2 und 3). Die genaue Anamnese ergab, daß der Patient die Symptome nach einer intensivmedizinischen Betreuung wegen eines Herzinfarktes ein halbes Jahr zuvor erstmalig bemerkt hatte. 


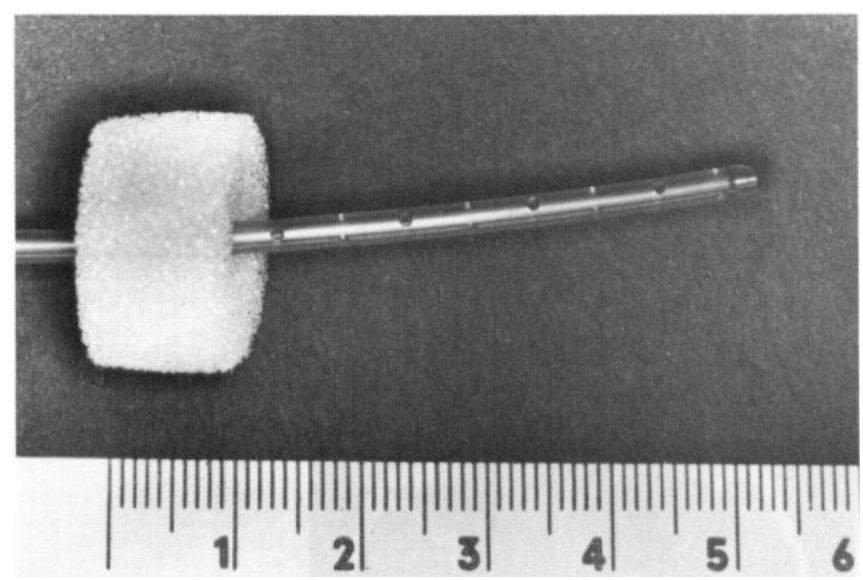

Abb.3 Die typische Schaumstoffummantelung einer Sauerstoffsonde, wie sie auf einer Intensivstation routinemäßig bei Herzinfarktpatienten in der ersten Behandlungsphase benutzt wird.

\section{Diskussion}

Ein derartiger Fall ist nach unserer Literaturübersicht bisher noch nicht beschrieben. Allerdings wurde 1983 am Klinikum Steglitz ein ähnliches Ereignis beobachtet.

Die Symptome einer blutig-eitrigen, streng einseitigen Rhinorrhoe lassen beim Kind zwar sofort an einen Nasenfremdkörper denken, bei einem 78 jährigen Mann ist dagegen die wichtigste Verdachtsdiagnose die eines (malignen) Nasentumors.
Für die Sicherung der richtigen Diagnose war im vorliegenden Fall nicht so sehr die Computertomographie, die die relativ grobe Struktur des Schaumstoffkörpers wegen des hohen Flüssigkeitsgehaltes nicht darstellen konnte, sondern vor allem die wiederholte Untersuchung mittels starrer Endoskopieoptiken entscheidend.

Da nicht auszuschließen ist, daß nach intensivmedizinischen Behandlungen häufiger eine Schaumstoffummantelung der Sauerstoffsonde in der Nase verbleibt, stellt sich die Frage, ob nicht durch Konstruktionsänderungen von seiten der Hersteller eine größere Sicherheit zu erzielen wäre. Zumindest wäre eine deutliche Röntgenmarkierung zu fordern, um ggf. den Fremdkörper mit Übersichtsaufnahmen leichter identifizierbar und lokalisierbar zu machen.

\section{Literatur}

1 Kindler, W.: Fremdkörper in der Nase und ihren Nebenhöhlen. In: Berendes, Link, Zöllner (Hrsg.): Hals-Nasen-Ohrenheilkunde, Band I. Thieme, Stuttgart, 1964

2 Legler, U.: Fremdkörper der Nase und ihrer Nebenhöhlen. In: Berendes, Link, Zöllner (Hrsg.): Hals-Nasen-Ohrenheilkunde in Praxis und Klinik, Band I, 2. Auflage. Thieme, Stuttgart, 1977

Dr. med. Jörg Ritter

Privatdozent Dr. med. Alexander Berghaus

HNO-Klinik des Universitätsklinikum Steglitz Berlin

Hindenburgdamm 30

D-1000 Berlin 45 